\title{
Sectoral Stock Prediction Using Convolutional Neural Networks with Candlestick Patterns as input Images
}

\author{
Artha Andriyanto ${ }^{1}$, Antoni Wibowo ${ }^{2}$, Norhaslinda Zainal Abidin ${ }^{3}$ \\ ${ }^{1}$ Computer Science Department, BINUS Graduate Program - Master of Computer Science, Bina Nusantara \\ University, Jakarta, Indonesia 11480, artha.andriyanto@binus.ac.id \\ ${ }^{2}$ Computer Science Department, BINUS Graduate Program - Master of Computer Science, Bina Nusantara \\ University, Jakarta, Indonesia 11480, anwibowo@binus.edu \\ ${ }^{3}$ Department of Decision Sciences, School of Quantitative Science - Universiti Utara Malaysia, \\ nhaslinda@uum.edu.my
}

\begin{abstract}
Stocks were one of the instruments for investment that could provide benefits rather than just saving money, but investments could also result in losses if wrong in investing in shares. Therefore determining the time frame beforehand will make easier to predict the trend of stock movements within a certain time period. Stock prediction analysis method which was quite popular among traders until now was to used candlesticks. Candlestick patterns were often used by stock prediction analysis in several centuries ago. The form of candlestick could infer the direction of prices. CNN, RNN and LTSM were currently widely used for forecasting in stock prediction movement. The most suitable method for image datasets was CNN. In this paper, we presented $\mathrm{CNN}$ and candlestick approach to recognize an image to identify the strength of a trend pattern in the prediction movement of stock. Based on our experiment, CNN with candlestick approach can produce up to $99.3 \%$ accuracy overall. Therefore, this method can produce good accuracy.
\end{abstract}

Key words : Candlestick, Convolution Neural Network, Deep learning, Stock Prediction, Technical Analysis.

\section{INTRODUCTION}

Every capital market participant usually requires an analytical tool to assist in making a decision to buy or sell a stock. Stock prediction in general has two methods, namely technical analysis and fundamental analysis [14]. Technical analysis is a prediction of stock movements with an approach in the form of a pattern of price movement charts while the fundamental analysis of the approach is to take into account the company's performance in financial statements, economic policies, certain commodities, political climate and so on. [1].

Analyst's recommendations stock with technical and fundamental analysis can obtain correct or incorrect decision to analyze the movements of a stock. The time frame on a stock becomes a parameter that greatly influences the trend of a stock. A stock can be said to be in an upward position when viewed from a time frame of 4 months or even years [2]. But stock prices on a weekly basis could have a down trend. Therefore, determining the time frame in advance will make it easier to predict the trend of stock movements within a certain time period. Various indicators are made to predict stocks such as MACD (Moving Average Convergence Divergence), a form of candlestick pattern, and external factors such as commodities and currencies [3]. The other approaches are Machine Learning [8] [13] and Deep Learning [9] [6] [10] [11].

In this paper, we want to combine $\mathrm{CNN}$ and candlestick approach to predict stock movements. CNN is used for image classification and recognition because of its high accuracy. It was proposed by computer scientist Yann LeCun in the late $90 \mathrm{~s}$, when he was inspired from the human visual perception of recognizing things [12]. CNN follows a hierarchical model that functions to build networks, such as the convolutional layer, max pooling, and finally provides a fully connected layer where all neurons are connected to each other and output is processed. The studied of image classification using deep learning, as in researched [15]. Researched on stock predictions had been conducted with an accuracy of 74.15\% [5]. Candlestick was an ancient charting method that emerged in japan and had proven its accuracy until now. By using the candlestick approached as a dataset was expected to obtain optimal prediction accuracy. The process of making a dataset will be categorized into an uptrend and downtrend, it was used as an input image to predict stock movements.

\section{REVIEW OF RELATED REVIEW}

Candlestick is a technical analysis method that was originally used by rice traders in Japan to observe and analyze the movement of rice prices in the market. A famous rice businessman from Japan in the 1700s named Munehisa Homma predicted the price movements of rice using routine notes such as opening, closing, highest, lowest prices on a 
daily basis. Based on these records, it produced a chart called a candlestick which was ultimately used by traders to predict prices at that time. There are two basic candlesticks as shown in Figure 1 namely (1) Bullish Candle when the closing price was higher than the opening price (usually green or white); (2) Bearish Candle when the lowest price is lower than the opening price (usually red or black) [4]. This study found that many single candlestick lines and reversal patterns can really help invest significantly positive mean rates of return by following candlestick trading strategies [7].

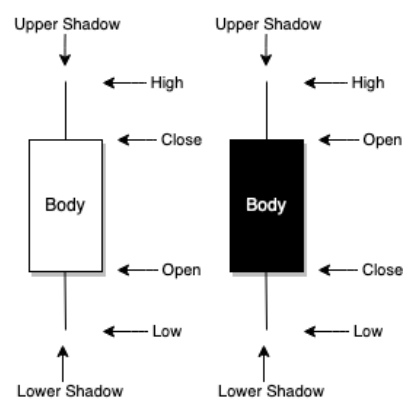

Figure 1: Model of candlestick

\subsection{Convolutional Neural Network (CNN)}

CNN was one of the deep learning algorithms that was usually used to analyze visual images. CNN architecture was similar to multilayer perceptron (MLP) which had several layers such as input, multiple hidden layers, and output to process data in the form of dimensions, in the process of deep learning the data will be used as training and testing. CNN had been widely used by researchers to solve various problems such as prediction, image recognition, classification and segmentation. Stock prediction research has been conducted using the Convolutional Neural Network. Such research is conducted by [5]. This study resulted in an accuracy of 74.15\% in Directional Accuracy (DA). Subsequent research conducted by [6]. The data set used consists of share prices every minute for 1721 companies registered on the NSE for the period July 2014 to June 2015. The results of the study have shown CNN provided more accurate results than RNN and LSTM models. Another conclusion, CNN can be identified as the best model for predicting stock movements using the sliding window model [6]. CNN was also used for prediction of film ratings by using several attributes of the film's historical values [16]. The next researched was the classification of copra meat done by developing a CNN algorithm that classifies the quality of copra [17].

\section{RESEARCH METHODOLOGY}

In this research, it takes several steps such as collecting data, making a dataset and the prediction process. The following flow in Figure 2 will be used as research. In the initial stage, the data will be collected first, then the data will be cleansed. After that the dataset is made by converting the daily stock price data into an image type in the form of candlesticks which data will be used as testing and training data. The training process will be conducted by experimenting with several epoch to get accurate predictions.

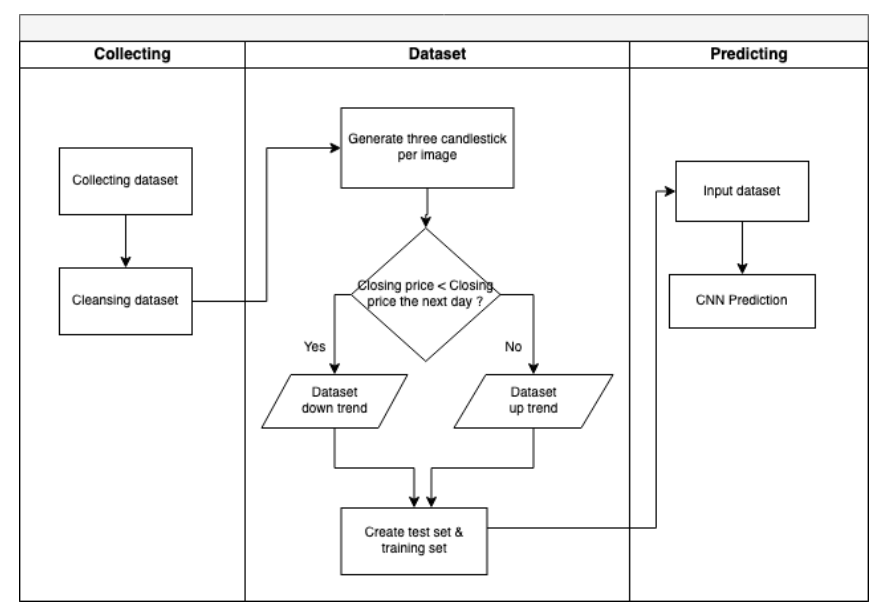

Figure 2: Flowchart for research

\subsection{Data Collection}

The initial stage of the research to be carried out is to collect data in advance. Data will be pulled from Yahoo financial site in the form of CSV. Index that will be used as research sampling for stock price prediction is the mining sector, namely the IDX Mining index (JKMING). Data will be taken from $01 / 01 / 2017$ to $30 / 12 / 2019$.

\subsection{Pre-Processing}

The dataset for training and testing will be produced with a total of three bars with a daily time frame. The results of the dataset generate are grouped into two categories up trend as shown in Figure 3 and down trend as shown in Figure 4. The dataset with the uptrend category is taken from the condition if the closing price on the third candlestick is smaller than the first candlestick in the next dataset while the dataset with the downtrend category is taken from the condition of the third candlestick with the closing price greater than the first candlestick on the next dataset. The below is the result of the generate dataset which will be used as training and testing to predict stock movements.

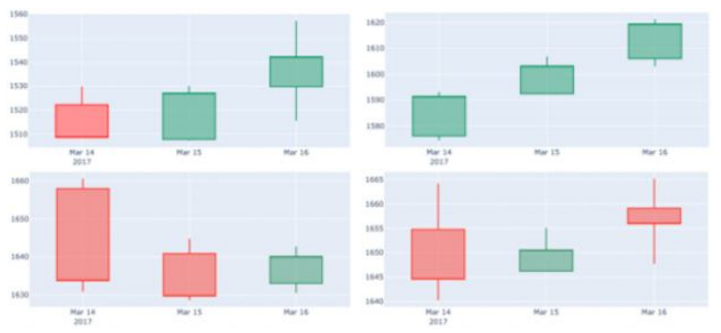

Figure 3: Candlestick up trend. 


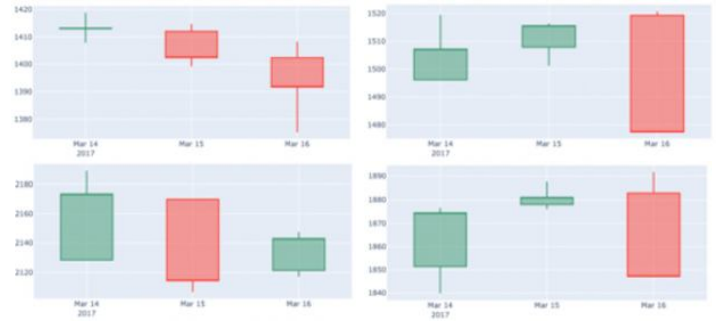

Figure 4: Candlestick down trend.

\subsection{Data Splitting}

To develop a prediction model, it is necessary to train the model and test the model. The dataset in model making is divided into $70-30 \%$ partitions. Seventy datasets will used to training data, and thirty percent will used for model testing. This is required for the process to determine performance of the prediction model.

\subsection{Convolutional Neural Network (CNN) architecture}

Table 1: CNN architecture.

\begin{tabular}{|c|}
\hline Conv2D 32, Relu \\
\hline MaxPooling2D \\
\hline Conv2D 48, Relu \\
\hline MaxPooling2D \\
\hline Flatten \\
\hline Dense(128, activation='relu')) \\
\hline $\begin{array}{c}\text { Dense(1, } \\
\text { activation='sigmoid') }\end{array}$ \\
\hline
\end{tabular}

The architecture of CNN was arranged in layers and patterns of connections between layers, activation functions, and learning methods. Neural network models and architecture determine how the process from input to output could influence the performance of the accuracy results. The architecture used in the studied was shown in Table 1, our CNN model architecture consist of 2 layers of convolutional 2D, 2 layers of max pooling 2D with pool size $(2,2)$, flatten and 2 dense with two activation relu and sigmoid.

\section{RESULTS AND DISCUSSIONS}

In this experiment, we used the $2.3 \mathrm{GHz}$ Intel Core i5 CPU with $8 \mathrm{~GB}$ of memory and the neural-network library using Keras, for training with steps per epoch 10, 20 and epochs 50, $100,200$.

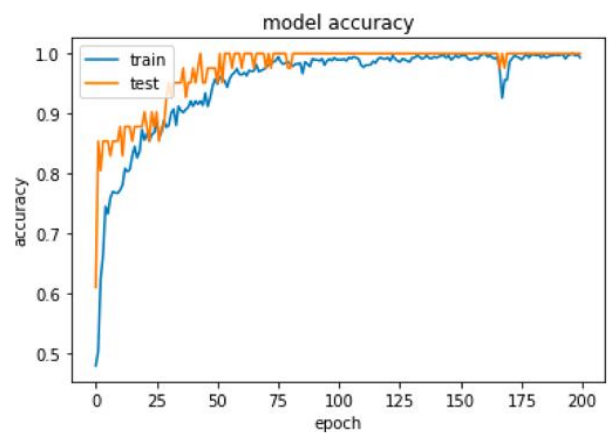

Figure 5: Result model accuracy

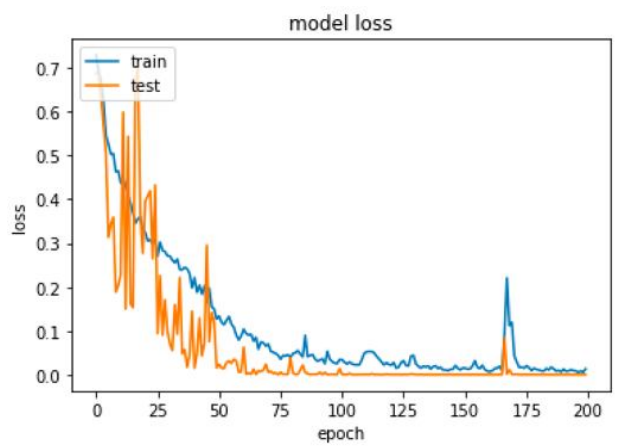

Figure 6: Result model loss

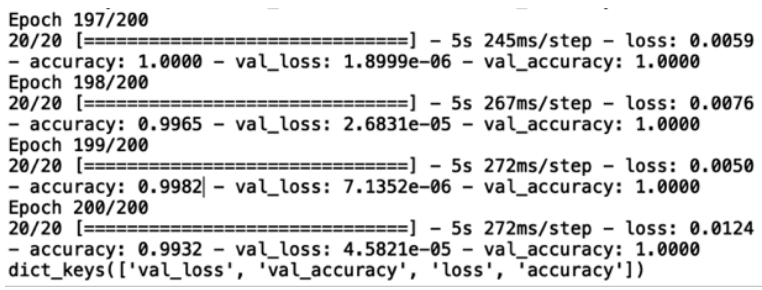

Figure 7: Result steps per epoch 20 and epochs 200

Figure 5 showed that the accuracy began to increase from the first epoch to 75 epoch, after that the accuracy stagnated at 0 . 99 until the end of the 200 epoch. However, there was an increase in the accuracy of the number of steps per epoch 20 and 100 epoch with steps per epoch 20 and 200 epoch that was an increase of 0.0143 . Figure 6 was a lost model figure that explains the value of loss will decrease if the number of epochs was increasing. Figure 7 showed the experiment with the steps per epoch of 20 and epochs 200 had the highest accuracy.

Table 2: Stock prediction training results

\begin{tabular}{|c|c|c|c|}
\hline $\begin{array}{c}\text { Steps per } \\
\text { epoch }\end{array}$ & epochs & loss & accuracy \\
\hline 10 & 50 & 0.2844 & 0.8682 \\
\hline 10 & 100 & 0.1675 & 0.9312 \\
\hline 10 & 200 & 0.0807 & 0.9696 \\
\hline
\end{tabular}




\begin{tabular}{|c|c|c|c|}
\hline 20 & 50 & 0.1046 & 0.9510 \\
\hline 20 & 100 & 0.0408 & 0.9789 \\
\hline 20 & 200 & 0.0124 & 0.9932 \\
\hline
\end{tabular}

From the experimental results in Table 2, we could conclude the greater the steps per epoch and epochs used in training will provide a higher prediction accuracy. From Table 2, we can see that CNN and candlestick approach with steps per epoch of 20 and 200 epochs provided an excellent accuracy rate of around $99.3 \%$.

\section{CONCLUSION}

In the paper we introduced $\mathrm{CNN}$ and candlestick approach to make stock predictions using CONV2D to process image data at the convolutional layer. The proposed method provided a quite good accuracy. Based on our experiment, CNN and candlestick approach with steps per epoch of 20 and 200 epochs provided an excellent accuracy rate of around $99.3 \%$.

\section{REFERENCES}

[1] Cohen, G., Kudryavtsev, A., \& Hon-Snir, S. (2011). Stock Market Analysis in Practice: Is It Technical or Fundamental? Journal of Applied Finance \& Banking, vol.1, pp 125-138.

[2] Shynkevicha, Y., McGinnity, T., Colemana, S. A., Belatrechec, A., \& Lid, Y. (2017). Forecasting Price Movements using Technical Indicators: Investigating the Impact of Varying Input Window Length. Neurocomputing, pp (71-88). https://doi.org/10.1016/j.neucom.2016.11.095

[3] Rajaa, S., \& Sahoo, J. K. (2019). Convolutional Feature Extraction and Neural Arithmetic Logic Units For Stock Prediction. arXiv.

[4] Joseph, G., Das, S. G., \& Romeo, A. (2015). A study on the formation of candlestick patterns with reference to nifty index for the past five years. Amrudha Romeo et al./ International Journal of Management Research \& Review, 67-90.

[5] Nino, J., evalo, A. A., Leon, D., Hernandez, G., \& Sandoval, J. S. (2018). Price Prediction with CNN and Limit Order Book Data. Workshop on Engineering Applications, WEA 2018, Medellín, Colombia. https://doi.org/10.1007/978-3-030-00350-0_11

[6] Selvin, S., R, V., E.A, G., Menon, V. K., \& K.P, S. (2017). Stock price prediction using LSTM, RNN and CNN-sliding window model. https://doi.org/10.1109/ICACCI.2017.8126078

[7] Goo, Y.-J., Chen, D.-H., \& C, Y.-W. (2007). The application of japanese candlestick trading strategies in taiwan. Investment Management and Financial Innovations, Vol. 4.
[8] Reddy, V. K. (2018). Stock Market Prediction Using Machine Learning. International Research Journal of Engineering and Technology (IRJET), Vol. 5.

[9] Vargas, M. R., Lima, B. S., \& Evsukoff, A. G. (2017). Deep learning for stock market prediction from financial news articles. IEEE.

[10] Wang, L., Lin, Z. Q., \& Wong, A. (2020). COVID-Net: A Tailored Deep Convolutional Neural Network Design for Detection of COVID-19 Cases from Chest X-Ray Images. arXiv.

[11] Sidra Mehtab, J. S. (2020). Stock Price Prediction Using Convolutional Neural Networks on a Multivariate Timeseries. arXiv.

[12] LeCun, Y., Bengio, Y., \& Hinton, G. (2015). Deep learning. Nature, Vol 521. https://doi.org/10.1038/nature14539

[13] Edgar P. Torres P.1(\&), M. H.-Á. (2019). Stock Market Data Prediction Using Machine Learning Techniques. Information Technology and Systems, pp.539-547. https://doi.org/10.1007/978-3-030-11890-7_52

[14] Wafia, A. S., Hassanb, H., \& Mabroukc, A. (2015). Fundamental Analysis Vs Technical Analysis in The Egyptian Stock Exchange - Empirical Study. International Journal of Business and Management Study - IJBMS, Volume 2.

[15] Krishna, M. M., Neelima, M., Harshali, M., \& Rao, M. V. (2018). Image classification using Deep learning. International Journal of Engineering \& Technology, 614-617. https://doi.org/10.14419/ijet.v7i2.7.10892

[16] Abarja, R. A., \& Wibowo, A. (2020). Movie Rating Prediction using Convolutional Neural Network based on Historical Values. IJETER, Volume 8. https://doi.org/10.30534/ijeter/2020/109852020

[17] Jr, R. I., Alon, A. S., Malbog, M. A., \& Gulmatico, J. S. (2020). Copra Meat Classification using Convolutional Neural Network. IJETER, Volume 8. https://doi.org/10.30534/ijeter/2020/30822020 